\title{
Asymptotic Behavior of SIRS Models in State-dependent Random Environments
}

\author{
Jianhai $\mathrm{Bao}^{b)}$, Jinghai Shao ${ }^{a)}$ \\ a) Center for Applied Mathematics, Tianjin University, Tianjin 300072, China \\ ${ }^{b)}$ Department of Mathematics, Swansea University, Singleton Park, SA2 8PP, UK \\ jianhaibao@csu.edu.cn, shaojh@tju.edu.cn
}

October 1, 2018

\begin{abstract}
The extinction and persistence of infective individuals are closely related to the random change of the environment. In this paper, via the random/stochastic SIRS models, we analyze qualitatively and quantitatively the impact caused by the random change of the environment. Our contributions consist in (i) giving some sufficient conditions on extinction (persistence) of the infectious individuals even though they are persistent (resp. extinct) in certain fixed environments; (ii) revealing the influence of random switching of incidence functions on extinction for the infectious individuals, which has not been studied before; (iii) establishing a criterion to judge extinction of the infectious individuals for a range of random/stochastic SIRS models with state-dependent switching via a stochastic comparison for functionals of jump processes. Moreover, some examples are set to illustrate the applications of our theory.
\end{abstract}

AMS subject Classification: 60J60, 65J05, 60H35

Key Words: regime-switching, extinction, persistence, state-dependence, stochastic comparison

\section{Introduction}

Let $S_{t}, I_{t}, R_{t}$ be the number of susceptible individuals, infective individuals, and removed individuals at time $t$, and $N_{t}=S_{t}+I_{t}+R_{t}$ be the totality of the population. Assume that infectious disease can cause additional mortality, and that an infectious individual can recover with a loss of immunity. Since the pioneer work due to Kermack-McKendrick [15], the SIRS model has been 
extensively investigated on, e.g., stability, extinction, persistence, Hopf bifurcation, to name a few. Different diseases have been discovered to be described via different incidence functions. So numerous variants of incidence rate functions have been put froward to fit well in the practical situation; see, e.g., 4, 9, 14, 15, 19, 25. In order to incorporate the effect of behavioral changes and prevent unbounded contact rates, [19] consider an SIRS model with a nonlinear incidence rate function in the form

$$
\left\{\begin{aligned}
\frac{\mathrm{d} S_{t}}{\mathrm{~d} t} & =\Lambda-\mu S_{t}-S_{t} G\left(I_{t}\right)+\gamma R_{t}, \\
\frac{\mathrm{d} I_{t}}{\mathrm{~d} t} & =S_{t} G\left(I_{t}\right)-(\mu+\nu+\delta) I_{t}, \\
\frac{\mathrm{d} R_{t}}{\mathrm{~d} t} & =\nu I_{t}-(\mu+\gamma) R_{t} .
\end{aligned}\right.
$$

The precise interpretations on the parameters in (1.1) are presented as follows: $\Lambda>0$ means the recruitment rate of the population; $\mu>0$ denotes the natural death rate of the population; $\delta$ stands for the disease inducing death rate; $\gamma>0$ signifies the rate at which recovered individuals lose immunity and return to the susceptible class; $\nu>0$ stipulates the natural recovery rate of the infectious individuals; $S G(\cdot)\left(G: \mathbb{R} \rightarrow \mathbb{R}_{+}\right)$manifests the incidence rate per infective individual. In particular, [19] initiated a nonlinear incidence function in the form

$$
G(x)=\frac{\beta x^{\ell}}{1+a x^{h}}, \quad x>0,
$$

where $\beta, \ell, h>0$ and $a \geq 0, \beta x^{\ell}$ measures the infection force of the disease and $1 /\left(1+a x^{h}\right)$ represents the inhibition effect from the behavioral change of the susceptible individuals when the number of infectious individuals increases. In (1.2), by taking $\ell=1$ and $a=0$, (1.1) goes back to an SIRS model with bilinear incidence rates (see e.g. [13, 15]). (1.1) is said to be the SIRS model with unbounded incidence function for $\ell>h$, saturated incidence function for $\ell=h$, and nonmonotone incidence function for $\ell<h$, respectively; see e.g. [14, 19, 22, 25, 29] and references within.

The deterministic SIRS models (1.1) have been extended in several different ways into stochastic or random counterparts. One of them is to perturb the deterministic models by white noises, see, for instance, [3, 16, 22, 26, 32] upon asymptotic analysis. Whereas, with regard to deterministic SIRS models or stochastic counterparts perturbed by white noises, the environment is assumed to be constant. As we know, the evolution of the diseases may heavily depend on the environment conditions such as temperature, humidity, etc. So, in practical situations, it is prerequisite to take the random changes of environmental conditions and their effects upon the spread of the disease into account, where one of natural and important questions is to justify the persistence or extinction of the disease. So, another extension of deterministic SIRS models is to perturb via the telegraph noises, which is, in general, called SIRS models with Markov 
switching or in random environments; see e.g. [11, 12, 14. For population dynamical systems in random environments, we refer to e.g. [2, 8, 10, 18].

In the present work we are interested in three kinds of SIRS models below.

Model I: Taking impacts of the random environments into consideration, we first consider the following state-independent regime-switching SIRS model:

$$
\left\{\begin{array}{l}
\frac{\mathrm{d}}{\mathrm{d} t} S_{t}=\Lambda_{\alpha_{t}}-\mu_{\alpha_{t}} S_{t}-G\left(I_{t}, \alpha_{t}\right) S_{t}+\gamma_{\alpha_{t}} R_{t} \\
\frac{\mathrm{d}}{\mathrm{d} t} I_{t}=G\left(I_{t}, \alpha_{t}\right) S_{t}-\left(\mu_{\alpha_{t}}+\nu_{\alpha_{t}}+\delta_{\alpha_{t}}\right) I_{t} \\
\frac{\mathrm{d}}{\mathrm{d} t} R_{t}=\nu_{\alpha_{t}} I_{t}-\left(\mu_{\alpha_{t}}+\gamma_{\alpha_{t}}\right) R_{t}
\end{array}\right.
$$

with the initial datum $\left(S_{0}, I_{0}, R_{0}\right)=\left(s_{0}, i_{0}, r_{0}\right) \in \mathbb{R}_{+}^{3}:=\left\{(x, y, z) \in \mathbb{R}^{3}: x>0, y>0, z>0\right\}$ and $\alpha_{0}=a_{0} \in \mathcal{M}=\{1,2, \ldots, M\}$ for some integer $M<\infty$. Herein, $\left(\alpha_{t}\right)_{t \geq 0}$ is a continuous-time Markov chain with the state space $\mathcal{M}$ and the transition probability specified by

$$
\mathbb{P}\left(\alpha_{t+\triangle}=j \mid \alpha_{t}=i\right)= \begin{cases}q_{i j} \triangle+o(\triangle), & i \neq j \\ 1+q_{i i} \triangle+o(\triangle), & i=j\end{cases}
$$

provided $\triangle \downarrow 0$ and inducing the $Q$-matrix $Q=\left(q_{i j}\right)_{i, j \in \mathcal{M}} ; G: \mathbb{R} \times \mathcal{M} \rightarrow \mathbb{R}_{+}$is continuous w.r.t. the first variable; $\mu_{i}, \gamma_{i}, \delta_{i}, \nu_{i}, i \in \mathcal{M}$, are positive constants, whose precise implications are explicated as in (1.1).

Model II: We are still interested in (1.3), whereas $\left(\alpha_{t}\right)_{t \geq 0}$ is a jump process with the state space $\mathcal{M}$ and the transition kernel stipulated as, for any $i, j \in \mathcal{M}$ and $x \in \mathbb{R}_{+}^{3}$,

$$
\mathbb{P}\left(\alpha_{t+\Delta}=j \mid \alpha_{t}=i, X_{t}=x\right)= \begin{cases}q_{i j}(x) \Delta+o(\Delta), & i \neq j \\ 1+q_{i i}(x) \Delta+o(\Delta), & i=j\end{cases}
$$

whenever $\Delta \downarrow 0$, where $X_{t}=\left(S_{t}, I_{t}, R_{t}\right) \in \mathbb{R}_{+}^{3}$.

Model III: Taking the influences of the state-dependent random environments and stochastic perturbations into account, we focus on the following SIRS model

$$
\left\{\begin{aligned}
\mathrm{d} S_{t} & =\left(\Lambda_{\alpha_{t}}-\mu_{\alpha_{t}} S_{t}-\frac{\beta_{\alpha_{t}} I_{t}}{f\left(I_{t}\right)} S_{t}+\gamma_{\alpha_{t}} R_{t}\right) \mathrm{d} t-\mu_{\alpha_{t}}^{e} S_{t} \mathrm{~d} B_{t}^{(1)}-\frac{\beta_{\alpha_{t}}^{e} I_{t}}{f\left(I_{t}\right)} S_{t} \mathrm{~d} B_{t}^{(2)} \\
\mathrm{d} I_{t} & =\left(\frac{\beta_{\alpha_{t}}^{e} S_{t}}{f\left(I_{t}\right)}-\left(\mu_{\alpha_{t}}+\nu_{\alpha_{t}}+\delta_{\alpha_{t}}\right)\right) I_{t} \mathrm{~d} t-\mu_{\alpha_{t}}^{e} I_{t} \mathrm{~d} B_{t}^{(1)}+\frac{\beta_{\alpha_{t}}^{e} S_{t}}{f\left(I_{t}\right)} I_{t} \mathrm{~d} B_{t}^{(2)} \\
\mathrm{d} R_{t} & =\left(\nu_{\alpha_{t}} I_{t}-\left(\mu_{\alpha_{t}}+\gamma_{\alpha_{t}}\right) R_{t}\right) \mathrm{d} t-\mu_{\alpha_{t}}^{e} R_{t} \mathrm{~d} B_{t}^{(1)}
\end{aligned}\right.
$$


with the initial datum $\left(S_{0}, I_{0}, R_{0}\right)=\left(s_{0}, i_{0}, r_{0}\right) \in \mathbb{R}_{+}^{3}$ and $\alpha_{0}=a_{0} \in \mathcal{M}$. Herein, $\mu_{i}^{e}, \beta_{i}^{e} \geq 0$; $f: \mathbb{R}_{+} \rightarrow \mathbb{R}_{+}$satisfies (A3) below; $B_{t}=\left(B_{t}^{(1)}, B_{t}^{(2)}\right)$ is a 2-dimensional Brownian motion defined on probability space $\left(\Omega, \mathscr{F},\left(\mathscr{F}_{t}\right)_{t \geq 0}, \mathbb{P}\right),\left(\alpha_{t}\right)_{t \geq 0}$ is a continuous time jump process determined by (1.5), and the other quantities are defined exactly as in (1.3).

Based on the three models above, in this work we aim to

(i) provide some sufficient conditions to guarantee the extinction (persistence) of the infectious individuals even though they are persistent (resp. extinct) in certain fixed environments;

(ii) illustrate the impacts of random switching of incidence functions on extinction for the infectious individuals;

(iii) establish a criterion to judge extinction of the infectious individuals for random/stochastic SIRS models with state-dependent regime switching.

Now we make the following remarks:

(1) The SIRS model (1.3) and (1.4) enjoys the following features: (i) It owns the incidence functions of one kind (e.g. $G(x, i)=\beta_{i} x / f(x)$ ), which however takes different values in different environments; (ii) It allows the incidence functions (e.g., $\left.G(x, i)=\frac{\beta_{i} x^{i}}{1+a x^{2}}\right)$ to be distinctive in different environments. See Theorems 2.5 and 2.8, corollaries [2.6, 2.7, 2.9, and Examples 2.1, 2.2, and 2.3 for more details.

(2) Compared with the SIRS model (1.3) and (1.4), there are essential challenges to cope with the model (1.3) and (1.5). For this setup, one of the challenges is that the classical ergodic theorem for continuous-time Markov chains does not work any more due to the fact that $\left(\alpha_{t}\right)_{t \geq 0}$ is merely a jump process rather than a Markov process. To get over such difficulty, we adopt a stochastic comparison approach (see Lemma 3.1 for further details) for functionals of the jump process $\left(\alpha_{t}\right)_{t \geq 0}$. Moreover, we provide explicit criteria on the extinction/persistence of the infectious individuals; see Theorems 3.2 and 3.3 and Examples 3.1 and 3.2 .

(3) Since the totality $N_{t}=S_{t}+I_{t}+R_{t}$ is variable, the approaches adopted to cope with (1.3) and (1.4) (or (1.5)) is unavailable for the model (1.6) and (1.5). So some tricks need to be put forward to investigate extinction of the infectious individuals; see Theorem 4.1 for further details.

The content of this paper is arranged as follows: Section 2 is concerned with impacts of state-independent random environments on existence and persistence for the infectious individuals solved by (1.3) and (1.4); Section 3 focuses on the influence of state-dependent random 
environments upon extinction/persistence of the infectious individuals determined by (1.3) and (1.5); Section 4 is devoted to extending the random SIRS model (1.3) and (1.4) (or (1.5)) into the stochastic counterpart (1.6) and (1.5) and providing some sufficient conditions to justify extinction of the infectious individuals.

\section{Impacts of state-independent random environments}

In the SIRS model (1.3) and (1.4), the transition rates of the continuous time Markov chain $\left(\alpha_{t}\right)_{t \geq 0}$ is state-independent. For related analysis of stochastic systems with state-independent random environments, we refer to e.g. [4, 8, 10, 11, 14, 17, 18, 21, 25] and references therein.

Let $\check{\Lambda}=\max _{i \in \mathcal{M}} \Lambda_{i}$ and $\hat{\Lambda}=\min _{i \in \mathcal{M}} \Lambda_{i}$. The other quantities $\check{\mu}, \hat{\mu}, \check{\beta}, \hat{\beta}, \cdots$, are defined analogously. Assume that

(A1) For each $i \in \mathcal{M}, G(\cdot, i): \mathbb{R} \rightarrow \mathbb{R}_{+}$is locally Lipschtz continuous and that there exists a constant $c>0$ such that $G(x, i) \leq c(1+|x|), x \in \mathbb{R}$;

(A2) The continuous-time Markov chain $\left(\alpha_{t}\right)$ is irreducible and positive recurrent with the invariant probability measure $\pi=\left(\pi_{1}, \cdots, \pi_{M}\right)$.

Remark 2.1 It is easy to check that the linear incidence rate (i.e., $G(x, i)=\beta_{i} x$ ), the saturated incidence rate (i.e., $G(x, i)=\frac{\beta_{i} x^{\ell}}{1+a x^{\ell}}, \ell>0$ ), the nonmonotone incidence rate (i.e., $G(x, i)=$ $\left.\frac{\beta_{i} x^{\ell}}{1+a x^{h}}, 0 \leq \ell<h\right)$, and the "media coverage" incidence rate (e.g., $\left.G(x, i)=\beta_{i} x \mathrm{e}^{-\alpha x}, \alpha>0\right)$ fulfill the assumption (A1) above.

The lemma below shows that the unique solution to (1.3) and (1.4) lies in the positive quadrant and implies that the totality of the population (i.e., $N_{t}$ ) has an upper bound.

Lemma 2.2 Assume that (A1) holds. Then, (1.3) and (1.4) has a unique strong solution $\left(S_{t}, I_{t}, R_{t}\right) \in \mathbb{R}_{+}^{3}$ with the initial value $\left(s_{0}, i_{0}, r_{0}\right) \in \mathbb{R}_{+}^{3}$. Moreover,

$$
N_{t} \leq N_{0} \mathrm{e}^{-\int_{0}^{t} \mu_{\alpha_{s}} \mathrm{~d} s}+\int_{0}^{t} \Lambda_{\alpha_{s}} \mathrm{e}^{-\int_{s}^{t} \mu_{\alpha_{r}} \mathrm{~d} r} \mathrm{~d} s, \quad \text { a.s. }
$$

Proof. The existence and uniqueness of positive solutions to (1.3) and (1.4) is more or less standard via a piecewise deterministic approach. Whereas, we herein provide a sketch of the proof to make the content self-contained.

Denote $0=\tau_{0}<\tau_{1}<\tau_{2}<\cdots<\tau_{n}<\cdots$ by the collection of all jump times of the Markov chain $\left(\alpha_{t}\right)_{t \geq 0}$. For any $t \in\left[0, \tau_{1}\right)$, under the assumption (A1), (1.3) with $\alpha_{t} \equiv \alpha_{0}$ has a 
unique strong solution $\left(S_{t}, I_{t}, R_{t}\right) \in \mathbb{R}_{+}^{3}$ by exploiting the Lyapunov function, for an appropriate constant $a>0$,

$$
V(x)=x_{1}-a-a \ln \left(x_{1} / a\right)+x_{2}-1-\ln x_{2}+x_{3}-1-\ln x_{3}>0, \quad x=\left(x_{1}, x_{2}, x_{3}\right) \in \mathbb{R}_{+}^{3}
$$

due to $y-1-\ln y>0$ for any $y>0$. In detail, please refer to the argument of e.g. [30, Theorem 3.1]). Next, for any $t \in\left[\tau_{1}, \tau_{2}\right)$, under the assumption (A1), (1.3) with $\alpha_{t} \equiv \alpha_{\tau_{1}}$ also admits a unique positive solution by adopting the same test function $V(x)$ above. Duplicating the previous procedure, we come to a conclusion that (1.3) enjoys a unique positive solution as for the initial value $\left(s_{0}, i_{0}, r_{0}\right) \in \mathbb{R}_{+}^{3}$.

Next, we aim to verify (2.1). From (1.3), we arrive at

$$
\mathrm{d} N_{t}=\left\{\Lambda_{\alpha_{t}}-\mu_{\alpha_{t}} N_{t}-\delta_{\alpha_{t}} I_{t}\right\} \mathrm{d} t, \quad t>0,
$$

which, along with $I_{t} \geq 0$, implies that

$$
\mathrm{d} N_{t} \leq\left\{\Lambda_{\alpha_{t}}-\mu_{\alpha_{t}} N_{t}\right\} \mathrm{d} t .
$$

This enables particularly us to obtain that

$$
\mathrm{d} N_{t} \leq\left\{\Lambda_{\alpha_{\tau_{k}}}-\mu_{\alpha_{\tau_{k}}} N_{t}\right\} \mathrm{d} t, \quad t \in\left[\tau_{k}, \tau_{k+1}\right), \quad k \in \mathbb{N} .
$$

Subsequently, the chain rule yields inductively that

$$
\begin{aligned}
N_{t} & \leq \mathrm{e}^{-\mu_{\alpha_{k}}\left(t-\tau_{k}\right)} N_{\tau_{k}}+\int_{\tau_{k}}^{t} \Lambda_{\alpha_{\tau_{k}}} \mathrm{e}^{-\mu_{\alpha_{k}}(t-s)} \mathrm{d} s \\
& =\mathrm{e}^{-\int_{\tau_{k}}^{t} \mu_{\alpha_{s}} \mathrm{~d} s} N_{\tau_{k}}+\int_{\tau_{k}}^{t} \Lambda_{\alpha_{s}} \mathrm{e}^{-\int_{s}^{t} \mu_{\alpha_{u}} \mathrm{~d} u} \mathrm{~d} s \\
& \leq \mathrm{e}^{-\int_{\tau_{k}}^{t} \mu_{\alpha_{s}} \mathrm{~d} s}\left\{\mathrm{e}^{-\int_{\tau_{k-1}}^{\tau_{k}} \mu_{\alpha_{s}} \mathrm{~d} s} N_{\tau_{k-1}}+\int_{\tau_{k-1}}^{\tau_{k}} \Lambda_{\alpha_{s}} \mathrm{e}^{-\int_{s}^{\tau_{k}} \mu_{\alpha_{u}} \mathrm{~d} u} \mathrm{~d} s\right\}+\int_{\tau_{k}}^{t} \Lambda_{\alpha_{s}} \mathrm{e}^{-\int_{s}^{t} \mu_{\alpha_{u}} \mathrm{~d} u} \mathrm{~d} s \\
& =\mathrm{e}^{-\int_{\tau_{k-1}}^{t} \mu_{\alpha_{s}} \mathrm{~d} s} N_{\tau_{k-1}}+\int_{\tau_{k-1}}^{t} \Lambda_{\alpha_{s}} \mathrm{e}^{-\int_{s}^{t} \mu_{\alpha_{u}} \mathrm{~d} u} \mathrm{~d} s \\
& \leq \cdots \\
& \leq \mathrm{e}^{-\int_{0}^{t} \mu_{\alpha_{s}} \mathrm{~d} s} N_{0}+\int_{0}^{t} \Lambda_{\alpha_{s}} \mathrm{e}^{-\int_{s}^{t} \mu_{\alpha_{u}} \mathrm{~d} u} \mathrm{~d} s .
\end{aligned}
$$

Whence, 2.1) is now available.

Remark 2.3 It seems that the assumption (A1) excludes the setting on unbounded incidence function. Concerning such setup, to verify the positive property of the solutions to (1.3) and (1.4) (or (1.5)), it is sufficient to follow the argument of Lemma 2.2 and combine with the cut-off approach. So Lemma 2.2 still holds whenever the assumption (A1) is replaced by (A1') below 
(A1') For each $i \in \mathcal{M}, G(\cdot, i): \mathbb{R} \rightarrow \mathbb{R}_{+}$is locally Lipschtz continuous and that there exist constants $c, k>0$ such that $G(x, i) \leq c\left(1+|x|^{k}\right), x \in \mathbb{R}$.

As a byproduct of Lemma 2.2, we derive that

Corollary 2.4 Under the assumption (A1), $\left(S_{t}, I_{t}, R_{t}, \alpha_{t}\right)_{t \geq 0}$ admits an invariant probability measure.

Proof. Remark that $\left(S_{t}, I_{t}, R_{t}, \alpha_{t}\right)_{t \geq 0}$ is a Feller process. According to (2.1), we deduce that

$$
S_{t} \leq N_{t} \leq N_{0} \mathrm{e}^{-\hat{\mu} t}+\check{\Lambda} / \hat{\mu} .
$$

For any $R>0$, let $B_{R}(0)=\left\{\left(s_{0}, i_{0}, r_{0}\right) \in \mathbb{R}_{+}^{3}: s_{0}+i_{0}+r_{0} \leq R\right\}$ and $P_{t}\left(s_{0}, i_{0}, r_{0}, i ; \cdot\right)$ be the transition kernel of $\left(S_{t}, I_{t}, R_{t}, \alpha_{t}\right)$ with the starting point $\left(s_{0}, i_{0}, r_{0}, i\right) \in \mathbb{R}_{+}^{3} \times \mathcal{M}$. For any $t>0$ and $\Gamma \in \mathscr{B}\left(\mathbb{R}_{+}^{3} \times \mathcal{M}\right)$, define the probability measure

$$
\mu_{t}(\Gamma)=\frac{1}{t} \int_{0}^{t} P_{s}\left(s_{0}, i_{0}, r_{0}, i ; \Gamma\right) \mathrm{d} s .
$$

Then, for any $\varepsilon>0$, by means of Chebyshev's inequality and (2.3), there exists an $R>0$ sufficiently large such that

$$
\mu_{t}\left(B_{R}(0) \times \mathcal{M}\right)=\frac{1}{t} \int_{0}^{t} P_{s}\left(s_{0}, i_{0}, r_{0}, i ; B_{R}(0) \times \mathcal{M}\right) \mathrm{d} s \geq 1-\frac{1}{R} \sup _{t \geq 0} \mathbb{E} N_{t} \geq 1-\varepsilon .
$$

Hence, $\left(\mu_{t}\right)_{t \geq 0}$ is tight since $B_{R}(0)$ is a compact subset of $\mathbb{R}_{+}^{3}$. As a result, $\left(S_{t}, I_{t}, R_{t}, \alpha_{t}\right)_{t \geq 0}$ admits an invariant probability measure via Krylov-Bogoliubov's theorem (see e.g. [7, Theorem 3.1.1]).

Our first main result in this paper is stated as below.

Theorem 2.5 Suppose (A1) and (A2) hold and assume further that there exist $\Phi:[0, \infty) \rightarrow$ $[0, \infty)$ with $\lim _{t \rightarrow \infty} \Phi_{t}=0$ and $\Upsilon: \mathcal{M} \rightarrow[0, \infty)$ such that

$$
G\left(I_{t}, \alpha_{t}\right) S_{t} / I_{t} \leq \Phi_{t}+\Upsilon_{\alpha_{t}}
$$

and that

$$
\Theta_{0}:=\frac{\sum_{i \in \mathcal{M}} \pi_{i} \Upsilon_{i}}{\sum_{i \in \mathcal{M}} \pi_{i}\left(\mu_{i}+\nu_{i}+\delta_{i}\right)}<1
$$

Then

$$
\lim _{t \rightarrow \infty} I_{t}=0, \quad \text { a.s. } \quad \text { and } \quad \lim _{t \rightarrow \infty} R_{t}=0, \quad \text { a.s. }
$$

and

$$
\lim _{t \rightarrow \infty}\left(\frac{1}{t} \int_{0}^{t} \mu_{\alpha_{s}} S_{s} \mathrm{~d} s\right)=\sum_{i \in \mathcal{M}} \pi_{i} \Lambda_{i}
$$


Proof. Keep $\left(S_{t}, I_{t}, R_{t}\right) \in \mathbb{R}_{+}^{3}$ in mind due to Lemma 2.2. From (1.3) and (2.4), it follows that

$$
\begin{aligned}
\frac{\mathrm{d}}{\mathrm{d} t} \ln I_{t} & =G\left(I_{t}, \alpha_{t}\right) S_{t} / I_{t}-\left(\mu_{\alpha_{t}}+\nu_{\alpha_{t}}+\delta_{\alpha_{t}}\right) \\
& \leq \Phi_{t}+\Upsilon_{\alpha_{t}}-\left(\mu_{\alpha_{t}}+\nu_{\alpha_{t}}+\delta_{\alpha_{t}}\right) .
\end{aligned}
$$

So one has

$$
\ln \left(I_{t} / I_{0}\right) \leq \int_{0}^{t} \Phi_{s} \mathrm{~d} s+\int_{0}^{t}\left\{\Upsilon_{\alpha_{s}}-\left(\mu_{\alpha_{s}}+\nu_{\alpha_{s}}+\delta_{\alpha_{s}}\right)\right\} \mathrm{d} s
$$

Hence, by virtue of the strong ergodicity theorem for Markov chains (see e.g. [1]), besides $\lim _{t \rightarrow \infty} \Phi_{t}=0$, we arrive at

$$
\limsup _{t \rightarrow \infty} \frac{\ln I_{t}}{t} \leq \sum_{i \in \mathcal{M}}\left\{\Upsilon_{i}-\left(\mu_{i}+\nu_{i}+\delta_{i}\right)\right\} \pi_{i}, \quad \text { a.s. }
$$

Thus, $\lim _{t \rightarrow \infty} I_{t}=0$, a.s., follows from (2.5).

In what follows, we intend to show $\lim _{t \rightarrow \infty} R_{t}=0$, a.s. To end this, observe that

$$
\mathrm{d} R_{t} \leq\left(\check{\nu} I_{t}-(\hat{\mu}+\hat{\gamma}) R_{t}\right) \mathrm{d} t
$$

Subsequently, by applying the chain rule to $\mathrm{d}\left(\mathrm{e}^{(\hat{\mu}+\hat{\gamma}) t} R_{t}\right)$, we deduce that

$$
R_{t} \leq R_{0} \mathrm{e}^{-(\hat{\mu}+\hat{\gamma}) t}+\check{\nu} \int_{0}^{t} \mathrm{e}^{-(\hat{\mu}+\hat{\gamma})(t-s)} I_{s} \mathrm{~d} s .
$$

Since $\lim _{t \rightarrow \infty} I_{t}=0$, a.s., for any $\varepsilon>0$, there exist $\Omega_{0} \subseteq \Omega$ with $\mathbb{P}\left(\Omega_{0}\right)=1$ and $T=T(\omega)>0$ such that

$$
I_{t}(\omega) \leq \varepsilon(\hat{\mu}+\hat{\gamma}) /(3 \check{\nu}), \quad t \geq T, \quad \omega \in \Omega_{0},
$$

which of course implies that

$$
\check{\nu} \int_{T}^{t} \mathrm{e}^{-(\hat{\mu}+\hat{\gamma})(t-s)} I_{s}(\omega) \mathrm{d} s \leq \varepsilon / 3, \quad \omega \in \Omega_{0}, \quad t \geq T .
$$

This, in addition to (2.10), yields that

$$
\begin{aligned}
R_{t}(\omega) & \leq R_{0} \mathrm{e}^{-(\hat{\mu}+\hat{\gamma}) t}+\check{\nu} \int_{0}^{T} \mathrm{e}^{-(\hat{\mu}+\hat{\gamma})(t-s)} I_{s}(\omega) \mathrm{d} s+\check{\nu} \int_{T}^{t} \mathrm{e}^{-(\hat{\mu}+\hat{\gamma})(t-s)} I_{s}(\omega) \mathrm{d} s \\
& \leq \varepsilon / 3+R_{0} \mathrm{e}^{-(\hat{\mu}+\hat{\gamma}) t}+\check{\nu}\left(N_{0}+\check{\Lambda} / \hat{\mu}\right) \int_{0}^{T} \mathrm{e}^{-(\hat{\mu}+\hat{\gamma})(t-s)} \mathrm{d} s \\
& \leq \varepsilon, \quad \omega \in \Omega_{0}
\end{aligned}
$$


for any

$$
t \geq T \vee\left(\frac{1}{\hat{\mu}+\hat{\gamma}} \ln \frac{3 R_{0}}{\varepsilon}\right) \vee\left(T+\frac{1}{\hat{\mu}+\hat{\gamma}} \ln \frac{3 \check{\nu}\left(N_{0}+\frac{\check{\Lambda}}{\hat{\mu}}\right)}{\varepsilon(\hat{\mu}+\hat{\gamma})}\right)
$$

Consequently, $\lim _{t \rightarrow \infty} R_{t}=0$, a.s., follows immediately.

By (2.6), one has

$$
\lim _{t \rightarrow \infty}\left(\frac{1}{t} \int_{0}^{t} I_{s} \mathrm{~d} s\right)=0, \quad \text { a.s. } \quad \text { and } \quad \lim _{t \rightarrow \infty}\left(\frac{1}{t} \int_{0}^{t} R_{s} \mathrm{~d} s\right)=0, \quad \text { a.s. }
$$

From (2.2), it follows that

$$
\frac{1}{t} \int_{0}^{t} \mu_{\alpha_{s}} S_{s} \mathrm{~d} s=\frac{N_{0}-N_{t}}{t}+\frac{1}{t} \int_{0}^{t}\left\{\Lambda_{\alpha_{s}}-\mu_{\alpha_{s}} R_{s}-\left(\mu_{\alpha_{s}}+\delta_{\alpha_{s}}\right) I_{s}\right\} \mathrm{d} s, \quad t>0 .
$$

This, in addition to (2.3), (2.11) as well as the strong ergodic theorem for the continuous-time Markov chains, yields the assertion (2.7).

It is easy to examine that all the incidence rate functions with $\ell>1$ satisfy (2.4) by taking advantage of Lemma 2.2. Now we present some applications of Theorem 2.5, Firstly, in (1.3) we choose

$$
G(x, i)=\beta_{i} x / f(x), \quad x \geq 0,
$$

where $\beta .: \mathcal{M} \rightarrow \mathbb{R}_{+}$and

(A3) $f: \mathbb{R}_{+} \rightarrow \mathbb{R}_{+}$with $f(0)=1$ is continuous and $f^{\prime}(x)>0$ for any $x \geq 0$.

The corollary below provides a sufficient criterion to examine the extinction of the infectious individuals even though the infectious individuals are persistent in some fixed environments.

Corollary 2.6 Let (A3) hold and assume that

$$
\Theta_{1}:=\frac{\check{\Lambda} \sum_{i \in \mathcal{M}} \pi_{i} \beta_{i}}{\hat{\mu} \sum_{i \in \mathcal{M}} \pi_{i}\left(\mu_{i}+\nu_{i}+\delta_{i}\right)}<1 .
$$

Then, for $\left(S_{t}, I_{t}, R_{t}\right)_{t \geq 0}$ solved by (1.3) and (1.4) with $G$ in (2.12), all of the assertions in Theorem 2.5 hold.

Proof. By $f(0)=1$ and $f^{\prime}(x)>0$ for any $x \geq 0$, we deduce that $G(x, i)=\beta_{i} x / f(x)$ satisfies the assumption (A1) so that Lemma 2.2 is applicable. From (2.3), together with $f(0)=1$ and $f^{\prime}(x)>0$ for any $x \geq 0$, we obtain that

$$
G\left(I_{t}, \alpha_{t}\right) S_{t} / I_{t}=\beta_{\alpha_{t}} S_{t} / f\left(I_{t}\right) \leq \beta_{\alpha_{t}} N_{t} \leq \beta_{\alpha_{t}}\left(N_{0} \mathrm{e}^{-\hat{\mu} t}+\check{\Lambda} / \hat{\mu}\right) .
$$


As a consequence, we infer that (2.4) holds with $\Phi_{t}=c \mathrm{e}^{-\hat{\mu} t}$ for some $c>0$ and $\Upsilon_{\alpha_{t}}=\check{\Lambda} \beta_{\alpha_{t}} / \hat{\mu}$ so that (2.5) is satisfied thanks to (2.13). Thus, the desired assertions follow from Theorem 2.5.

Another application of Theorem 2.5 is to take

$$
G(x, i)=\frac{\beta_{i} x^{i}}{1+a x^{2}}, \quad x \geq 0
$$

for some $\beta$. $: \mathcal{M} \rightarrow \mathbb{R}_{+}$and $a>0$. The following corollary reveals the influence of the random switching of the incidence functions on extinction of infectious individuals.

Corollary 2.7 Let (A2) hold and assume that

$$
\Theta_{2}:=\frac{\sum_{i \in \mathcal{M}} \pi_{i} \beta_{i}(\check{\Lambda} / \hat{\mu})^{i}}{\sum_{i \in \mathcal{M}} \pi_{i}\left(\mu_{i}+\nu_{i}+\delta_{i}\right)}<1,
$$

then, for $\left(S_{t}, I_{t}, R_{t}\right)_{t \geq 0}$ solved by (1.3) and (1.4) with $G$ in (2.14), all of the assertions in Theorem 2.5 hold.

Proof. In terms of the definition of $G$ introduced in (2.14) and by taking Remark 2.3 into account, we deduce that $0<I_{t}, S_{t} \leq N_{t}$ and (2.3) holds so that

$$
\begin{aligned}
G\left(I_{t}, \alpha_{t}\right) S_{t} / I_{t} & =\frac{\beta_{\alpha_{t}} I_{t}^{\alpha_{t}-1} S_{t}}{1+a I_{t}^{2}} \leq \beta_{\alpha_{t}} N_{t}^{\alpha_{t}}=\beta_{\alpha_{t}} \sum_{i=1}^{M} N_{t}^{i} \mathbf{1}_{\left\{\alpha_{t}=i\right\}} \\
& \leq \beta_{\alpha_{t}} \sum_{i=1}^{M}\left(N_{0} \mathrm{e}^{-\hat{\mu} t}+\check{\Lambda} / \hat{\mu}\right)^{i} \mathbf{1}_{\left\{\alpha_{t}=i\right\}} \\
& \leq c \mathrm{e}^{-\hat{\mu} t}+\beta_{\alpha_{t}} \sum_{i=1}^{M}(\check{\Lambda} / \hat{\mu})^{i} \mathbf{1}_{\left\{\alpha_{t}=i\right\}} \\
& =c \mathrm{e}^{-\hat{\mu} t}+\beta_{\alpha_{t}}(\check{\Lambda} / \hat{\mu})^{\alpha_{t}}
\end{aligned}
$$

for some constant $c>0$. Therefore, (2.4) holds with $\Phi_{t}=c \mathrm{e}^{-\hat{\mu} t}$ and $\Upsilon_{\alpha_{t}}=\beta_{\alpha_{t}}(\check{\Lambda} / \hat{\mu})^{\alpha_{t}}$. On the other hand, (2.5) follows owing to (2.15). As a result, all of the assertions hold true in terms of Theorem 2.5 .

Now we proceed to provide some examples to illustrate the applications of Corollaries 2.6 and 2.7 so that our main result (i.e. Theorem 2.5) is applicable. To portray the behavior of the infectious individuals in each fixed environment, we introduce the quantity $R_{0}^{(i)}, i \in \mathcal{M}$, defined by

$$
R_{0}^{(i)}=\frac{\Lambda_{i} \beta_{i}}{\mu_{i}\left(\mu_{i}+\nu_{i}+\delta_{i}\right)}, \quad i \in \mathcal{M}
$$


Example 2.1 We focus on the model (1.3), in which $G$ is given as in (2.12) with $f(0)=1$, and $\left(\alpha_{t}\right)_{t \geq 0}$ is a continuous-time Markov chain with the state space $\mathcal{M}=\{1,2\}$ and the $Q$-matrix

$$
Q=\left(\begin{array}{cc}
-p & p \\
q & -q
\end{array}\right), \quad p, q>0
$$

Assume that

$$
\begin{gathered}
\Lambda_{2}<\Lambda_{1}, \quad \mu_{2}<\mu_{1}, \\
\left(\mu_{1}+\nu_{1}+\delta_{1}\right) / \beta_{1}>\Lambda_{1} / \mu_{2}, \quad\left(\mu_{2}+\nu_{2}+\delta_{2}\right) / \beta_{2}<\Lambda_{2} / \mu_{2},
\end{gathered}
$$

and that

$$
\frac{q}{p}>\frac{\Lambda_{1} \beta_{2}-\mu_{2}\left(\mu_{2}+\nu_{2}+\delta_{2}\right)}{\mu_{2}\left(\mu_{1}+\nu_{1}+\delta_{1}\right)-\Lambda_{1} \beta_{1}}>0
$$

It is apparent that (2.19) implies that $\check{\Lambda}=\Lambda_{1}$ and $\hat{\mu}=\mu_{2}$. By a simple calculation, the unique invariant probability measure of $\left(\alpha_{t}\right)_{t \geq 0}$ is given by

$$
\pi=\left(\pi_{1}, \pi_{2}\right)=\left(\frac{q}{p+q}, \frac{p}{p+q}\right) .
$$

Hence, by taking (2.21) into consideration, it follows that

$$
\Theta_{1}=\frac{\Lambda_{1}\left(q \beta_{1}+p \beta_{2}\right)}{\mu_{2}\left\{q\left(\mu_{1}+\nu_{1}+\delta_{1}\right)+p\left(\mu_{2}+\nu_{2}+\delta_{2}\right)\right\}}<1 .
$$

Whence, Corollary 2.6 implies $\lim _{t \rightarrow \infty} I_{t}=0$, a.s., and, $\lim _{t \rightarrow \infty} R_{t}=0$, a.s.

In view of $f(0)=1$ and (2.20), one has $R_{0}^{(1)}<1, R_{0}^{(2)}>1$. As a result, in terms of [3, Theorem 2.1], the disease-free equilibrium $E_{0}^{(1)}:=\left(\Lambda_{1} / \mu_{1}, 0,0\right)$ of the SIRS model (1.3) with $\alpha_{t} \equiv$ 1 is globally asymptotically stable, whereas the disease-free equilibrium $E_{0}^{(2)}:=\left(\Lambda_{2} / \mu_{2}, 0,0\right)$ of the SIRS model (1.3) with $\alpha_{t} \equiv 2$ is unstable. Obviously, (2.20) and (2.21) hold for

$$
\beta_{1}=\mu_{2}=\nu_{2}=\Lambda_{2}=0.1, \mu_{1}=\nu_{1}=p=0.2, \beta_{2}=0.3, \delta_{1}=0.05, \delta_{2}=0.04, \Lambda_{1}=0.4, q=4 .
$$

The following example shows that the random switching of the incidence functions can improve the extinction of the infectious individuals in certain sense. More precisely, for the model (1.3) with $G$ given in (2.14), the infectious individuals are extinct although they might be persistent with certain incidence function in some environment.

Example 2.2 Consider the model (1.3) with $G$ introduced in (2.14), where $\left(\alpha_{t}\right)$ is a continuoustime Markov chain with the state space $\mathcal{M}=\{1,2\}$ and the $Q$-matrix $Q$ given by (2.18). Assume that

$$
\Lambda_{2}<\Lambda_{1}, \quad \mu_{2}<\mu_{1}
$$




$$
\beta_{2}\left(\frac{\Lambda_{1}}{\mu_{2}}\right)^{2}<\mu_{2}+\nu_{2}+\delta_{2}, \quad \mu_{1}\left(\mu_{1}+\nu_{1}+\delta_{1}\right)<\beta_{1} \Lambda_{1}
$$

and that

$$
0<\frac{q}{p}<\frac{\mu_{2}+\nu_{2}+\delta_{2}-\beta_{2}\left(\frac{\Lambda_{1}}{\mu_{2}}\right)^{2}}{\frac{\beta_{1} \Lambda_{1}}{\mu_{2}}-\left(\mu_{1}+\nu_{1}+\delta_{1}\right)} .
$$

Thanks to (2.23), one has $\check{\Lambda}=\Lambda_{1}$ and $\hat{\mu}=\mu_{2}$. On the other hand, it is easy to see that $\Theta_{2}$, defined in (2.15), is less than 1 by virtue of (2.25). Hence, Corollary 2.7 demonstrates that $\lim _{t \rightarrow \infty} I_{t}=0$, a.s., and, $\lim _{t \rightarrow \infty} R_{t}=0$, a.s.

Also, it follows from (2.24) that $R_{0}^{(2)}$, defined in (2.17), is greater than 1. Consequently, $I_{t}$ with $\alpha_{t} \equiv 1$ is unstable due to [3, Theorem 2.1]. More concretely, by taking

$$
\left\{\begin{array} { l } 
{ \Lambda _ { 1 } = 0 . 4 } \\
{ \Lambda _ { 2 } = 0 . 3 }
\end{array} \quad \left\{\begin{array} { l } 
{ \mu _ { 1 } = 0 . 2 5 } \\
{ \mu _ { 2 } = 0 . 2 }
\end{array} \quad \left\{\begin{array} { l } 
{ \beta _ { 1 } = 0 . 3 } \\
{ \beta _ { 2 } = 0 . 1 }
\end{array} \quad \left\{\begin{array} { l } 
{ \nu _ { 1 } = 0 . 2 } \\
{ \nu _ { 2 } = 0 . 3 }
\end{array} \quad \left\{\begin{array} { l } 
{ \delta _ { 1 } = 0 . 0 2 } \\
{ \delta _ { 2 } = 0 . 0 5 }
\end{array} \quad \left\{\begin{array}{l}
p=3 \\
q=2
\end{array},\right.\right.\right.\right.\right.\right.
$$

we find that (2.23)-(2.25) hold, respectively.

The following theorem presents some sufficient conditions to depict the persistence of the infectious individuals. The criterion provided allows the infectious individuals to be extinct in certain environments.

Theorem 2.8 Let (A1) and (A2) hold and suppose further that $\lim _{x \rightarrow 0} G(x, j) / x>0$ for any $j \in \mathcal{M}$ and that

$$
\Theta_{3}:=\frac{\sum_{i \in \mathcal{M}} \pi_{i} \Lambda_{i}}{\tau \sum_{i \in \mathcal{M}} \pi_{i}\left(\mu_{i}+\nu_{i}+\delta_{i}\right)}>1,
$$

where

$$
\tau:=\max _{j \in \mathcal{M}}\left(\frac{G(0, j)+\mu_{j}}{\lim _{x \rightarrow 0} G(x, j) / x}\right) .
$$

Then

$$
\liminf _{t \rightarrow \infty}\left(\frac{1}{t} \int_{0}^{t} I_{s} \mathrm{~d} s\right)>0, \quad \text { a.s., }
$$

that is, the infectious individuals is persistent.

Proof. First of all, we claim that there exists a constant $K>0$ such that

$$
F_{j, y}(x):=K x+\left((\tau / x-1) G(x, j)-\mu_{j}\right) y \geq 0
$$

for any $0 \leq x, y \leq N_{0}+\check{\Lambda} / \hat{\mu}$ and $j \in \mathcal{M}$. Obviously, (2.29) holds whenever $y=0$. So, it is sufficient to verify that (2.29) holds for $0<y \leq N_{0}+\check{\Lambda} / \hat{\mu}$. In what follows, we set $0<y \leq$ $N_{0}+\check{\Lambda} / \hat{\mu}$. According to the definition of $\tau$, it is obvious to see that

$$
F_{j, y}(0)=\left(\tau \lim _{x \rightarrow 0} G(x, j) / x-G(0, j)-\mu_{j}\right) y>0 .
$$


By the continuity of $x \mapsto F_{j, y}(x)$, we deduce from (2.30) that there exists $0<x_{0}<N_{0}+\check{\Lambda} / \hat{\mu}$ such that (2.29) holds for some $K=K_{0}>0$ and any $x \in\left[0, x_{0}\right]$. Next, for any $x \in\left[x_{0}, N_{0}+\check{\Lambda} / \hat{\mu}\right]$, observe that

$$
F_{j, y}(x) \geq K x_{0}-\max _{x \in\left[x_{0}, N_{0}+\check{\Lambda} / \hat{\mu}\right], j \in \mathcal{M}}\left|(\tau / x-1) G(x, j)-\mu_{j}\right|\left(N_{0}+\check{\Lambda} / \hat{\mu}\right) .
$$

Thus, (2.29) is available by taking $K>0$ sufficiently large.

Taking advantage of $R_{t} \geq 0$, a.s., we infer that

$$
\begin{aligned}
\frac{\mathrm{d}}{\mathrm{d} t} S_{t} & \geq \Lambda_{\alpha_{t}}-\mu_{\alpha_{t}} S_{t}-G\left(I_{t}, \alpha_{t}\right) S_{t} \\
& =\Lambda_{\alpha_{t}}-\tau G\left(I_{t}, \alpha_{t}\right) S_{t} / I_{t}-K I_{t}+F_{\alpha_{t}, S_{t}}\left(I_{t}\right) \\
& \geq \Lambda_{\alpha_{t}}-\tau G\left(I_{t}, \alpha_{t}\right) S_{t} / I_{t}-K I_{t},
\end{aligned}
$$

which further yields that

$$
\tau \int_{0}^{t} \frac{G\left(I_{s}, \alpha_{s}\right) S_{s}}{I_{s}} \mathrm{~d} s \geq S_{0}-S_{t}-K \int_{0}^{t} I_{s} \mathrm{~d} s+\int_{0}^{t} \Lambda_{\alpha_{s}} \mathrm{~d} s
$$

Substituting the first display of (2.8) into (2.32) and taking (2.3) into account, one has

$$
K \int_{0}^{t} I_{s} \mathrm{~d} s \geq S_{0}-N_{0}-\check{\Lambda} / \hat{\mu}-\tau \ln c+\int_{0}^{t}\left(\Lambda_{\alpha_{s}}-\tau\left(\mu_{\alpha_{s}}+\nu_{\alpha_{s}}+\nu_{\alpha_{s}}\right)\right) \mathrm{d} s
$$

for some constant $c>0$. Consequently, the strong ergodicity theorem for the Markov chain $\left(\alpha_{t}\right)_{t \geq 0}$ yields that

$$
K \liminf _{t \rightarrow \infty}\left(\frac{1}{t} \int_{0}^{t} I_{s} \mathrm{~d} s\right) \geq \sum_{j \in \mathcal{M}}\left(\Lambda_{j}-\tau\left(\mu_{j}+\nu_{j}+\delta_{j}\right)\right) \pi_{j} .
$$

Whence, (2.28) follows directly from (2.26).

Applying Theorem 2.8 to the incidence rate function $G$ in (2.12), we obtain the following corollary, which states some sufficient conditions to examine the persistence of the individuals.

Corollary 2.9 Let (A2) hold and suppose further that

$$
\Theta_{4}:=\frac{\sum_{i \in \mathcal{M}} \pi_{i} \Lambda_{i}}{\max _{i \in \mathcal{M}}\left(\mu_{i} / \beta_{i}\right) \sum_{i \in \mathcal{M}} \pi_{i}\left(\mu_{i}+\nu_{i}+\delta_{i}\right)}>1
$$

Then

$$
\liminf _{t \rightarrow \infty}\left(\frac{1}{t} \int_{0}^{t} I_{s} \mathrm{~d} s\right)>0, \quad \text { a.s. }
$$


Proof. By the structure of $G$ given in (2.12), we have $G(0, j)=0$ and $\lim _{x \rightarrow 0} G(x, j) / x=\beta_{j}$ due to $f(0)=1$ so that $\tau=\max _{i \in \mathcal{M}}\left(\mu_{i} / \beta_{i}\right)$. With (2.34) in hand, we therefore infer that (2.26) holds. Accordingly, the desired assertion (2.35) is verified.

Below, let's revisit Example 2.1 which, under certain appropriate conditions, illustrates that the infectious individuals is persistent although they might die out in some environments.

Example 2.3 Let's reconsider Example 2.1. Assume that

$$
\frac{\mu_{2}+\nu_{2}+\delta_{2}}{\Lambda_{2}}<\frac{\beta_{1}}{\mu_{1}}<\frac{\beta_{2}}{\mu_{2}} \wedge \frac{\mu_{1}+\nu_{1}+\delta_{1}}{\Lambda_{1}}
$$

and that

$$
0<\frac{q}{p}<\frac{\beta_{1} \Lambda_{2}-\mu_{1}\left(\mu_{2}+\nu_{2}+\delta_{2}\right)}{\mu_{1}\left(\mu_{1}+\nu_{1}+\delta_{1}\right)-\beta_{1} \Lambda_{1}}
$$

In accordance with (2.22) and (2.36), $\Theta_{3}$, introduced in (2.26), reads as follows

$$
\Theta_{3}=\frac{q \Lambda_{1}+p \Lambda_{2}}{\frac{\mu_{1}}{\beta_{1}}\left\{q\left(\mu_{1}+\nu_{1}+\delta_{1}\right)+p\left(\mu_{2}+\nu_{2}+\delta_{2}\right)\right\}}<1 .
$$

Thus, with the help of Corollary 2.9, we arrive at

$$
\liminf _{t \rightarrow \infty}\left(\frac{1}{t} \int_{0}^{t} I_{s} \mathrm{~d} s\right)>0, \quad \text { a.s. }
$$

Nevertheless, by virtue of (2.36), it follows that $R_{0}^{(1)}<1$ and $R_{0}^{(2)}>1$ such that $\lim _{t \rightarrow \infty} I_{t}=0$ (for the case $\alpha_{t} \equiv 1$ ), a.s., and $\lim _{t \rightarrow \infty} I_{t}>0$ (for the case $\alpha_{t} \equiv 2$ ), a.s. So, the infectious individuals is persistent in the environment 1 and dies out in the environment 2.

The corollary below explicates that the assumptions imposed in Corollaries 2.6] and 2.9] are compatible.

Corollary 2.10 It holds $\Theta_{4} \leq \Theta_{1}$, and further $\Theta_{4}=\Theta_{1}$ if and only if $\Lambda_{i}, \beta_{i}$, $\mu_{i}$ are all independent of $i$, i.e., for some positive constants $\Lambda, \beta, \mu, \Lambda_{i} \equiv \Lambda, \beta_{i} \equiv \beta, \mu_{i} \equiv \mu, i \in \mathcal{M}$.

Proof. According to the notions of $\Theta_{1}$ and $\Theta_{4}$, we deduce that

$$
\frac{\Theta_{4}}{\Theta_{1}}=\frac{\sum_{i \in \mathcal{M}} \pi_{i}\left(\frac{\Lambda_{i}}{\bar{\Lambda}}\right)}{\sum_{i \in \mathcal{M}} \pi_{i} \beta_{i} \max _{j \in \mathcal{M}}\left(\frac{\mu_{j}}{\hat{\mu}} \cdot \frac{1}{\beta_{j}}\right)} \leq \frac{\sum_{i \in \mathcal{M}} \pi_{i}\left(\frac{\Lambda_{i}}{\hat{\Lambda}}\right)}{\sum_{i \in \mathcal{M}} \pi_{i}\left(\frac{\beta_{i}}{\hat{\beta}}\right)} \leq 1,
$$

that is, $\Theta_{4} \leq \Theta_{1}$. It is obvious to observe that $\Theta_{4}=\Theta_{1}$ whenever $\Lambda_{i}, \beta_{i}$ and $\mu_{i}$ are constant. Now, if $\Theta_{4}=\Theta_{1}$, in view of the first inequality in (2.37), we have $\check{\Lambda}=\Lambda_{i}$ and $\hat{\beta}=\beta_{i}, i \in \mathcal{M}$, 
namely, both $\Lambda_{i}$ and $\beta_{i}$ are constant. Whence, exploiting the identity in (4.19), we arrive at $\check{\beta}=\hat{\beta}$, which further means that $\beta_{i}, i \in \mathcal{M}$, is independent of the index $i$.

In Examples 2.1] and 2.3, we explain that the infectious individuals are extinct (resp. persistent) although they might persist (resp. die out) in some environments. Yet one may be quite interested in the examples, where the infectious individuals are extinct (resp. persistent) even though the infectious individuals are persistent (resp. extinct) in each fixed environment. Nevertheless, the following corollary shows that the scenario mentioned cannot take place.

Corollary 2.11 Let (A2) hold.

(i) If, for each $i \in \mathcal{M}, R_{0}^{(i)} \leq 1$, then it always holds $\Theta_{4} \leq 1$ whatever the irreducible transition rate matrix of the random switching process $\left(\alpha_{t}\right)_{t \geq 0}$ is.

(ii) If, for each $i \in \mathcal{M}, R_{0}^{(i)}>1$, then it always hold $\Theta_{4}>1$ whatever the irreducible transition rate matrix of the random switching process $\left(\alpha_{t}\right)_{t \geq 0}$ is.

Proof. By the definition of $R_{0}^{(i)}$ introduced in (2.17), one has $\mu_{i}+\nu_{i}+\delta_{i}=\frac{\Lambda_{i} \beta_{i}}{\mu_{i} R_{0}^{(i)}}$. Then, $\Theta_{0}$ and $\Theta_{1}$ can be reformulated, respectively, as

$$
\Theta_{1}=\frac{\check{\Lambda} \sum_{i \in \mathcal{M}} \pi_{i} \beta_{i}}{\hat{\mu} \sum_{i \in \mathcal{M}} \pi_{i} \frac{\Lambda_{i} \beta_{i}}{\mu_{i} R_{0}^{(i)}}} \quad \text { and } \quad \Theta_{4}=\frac{\sum_{i \in \mathcal{M}} \pi_{i} \Lambda_{i}}{\max _{i \in \mathcal{M}}\left(\frac{\mu_{i}}{\beta_{i}}\right) \sum_{i \in \mathcal{M}} \pi_{i} \frac{\Lambda_{i} \beta_{i}}{\mu_{i} R_{0}^{(i)}}} .
$$

If $R_{0}^{(i)} \leq 1$ for each $i \in \mathcal{M}$, then

$$
\Theta_{4}=\frac{\sum_{i \in \mathcal{M}} \pi_{i} \Lambda_{i}}{\sum_{i \in \mathcal{M}} \pi_{i} \Lambda_{i} \cdot \frac{\beta_{i} / \mu_{i}}{\min _{j \in \mathcal{M}}\left(\beta_{j} / \mu_{j}\right)} \cdot \frac{1}{R_{0}^{(i)}}} \leq 1,
$$

owing to $\frac{\beta_{i} / \mu_{i}}{\min _{j \in \mathcal{M}}\left(\beta_{j} / \mu_{j}\right)} \geq 1$ and $1 / R_{0}^{(i)} \geq 1$. This gives us the assertion (i). Next, in case of $R_{0}^{(i)}>1$, it follows from $\Lambda_{i} / \check{\Lambda} \leq 1$ and $\hat{\mu} / \mu_{i} \leq 1$ that

$$
\Theta_{1}=\frac{\sum_{i \in \mathcal{M}} \pi_{i} \beta_{i}}{\sum_{i \in \mathcal{M}} \pi \beta_{i} \frac{\Lambda_{i}}{\Lambda} \cdot \frac{\hat{\mu}}{\mu_{i}} \cdot \frac{1}{R_{0}^{(i)}}}>1
$$

which further yields the desired conclusion (ii).

\section{Impacts of state-dependent random environments}

In this section, we move forward to deal with impacts of state-dependent random environments upon extinction and persistence of the infectious individuals. As an illustrative work, in this 
part we are interested in the SIRS model (1.3) and (1.5). As we know, (1.3) and (1.5) is a kind of state-dependent regime switching diffusions, which have been investigated considerably on e.g. stability, ergodicity and numerical approximation in the past decade; see e.g. [23, 28, 31] and references therein. It is worthy to point out that the quadruple $\left(S_{t}, I_{t}, R_{t}, \alpha_{t}\right)_{t \geq 0}$ is a Markov process although neither $\left(S_{t}, I_{t}, R_{t}\right)_{t \geq 0}$ nor $\left(\alpha_{t}\right)_{t \geq 0}$ is. Assume further that

(Q1) For each $x \in \mathbb{R}_{+}^{3}$, the matrix $Q(x)=\left(q_{i j}(x)\right)_{i, j \in \mathcal{M}}$ is irreducible and conservative,

(Q2) $H:=\sup _{x \in \mathbb{R}_{+}^{3}} \max _{i \in \mathcal{M}} q_{i}(x)<\infty$, where $q_{i}(x):=\sum_{j \neq i} q_{i j}(x)$.

In contrast to the SIRS model (1.3) and (1.4), there are essential challenges to cope with the model (1.3) and (1.5). For this setup, one of the challenges is that the classical ergodic theorem for continuous-time Markov chains does not work any more due to the fact that $\left(\alpha_{t}\right)_{t \geq 0}$ is merely a jump process rather than a Markov process. To get over such difficulty, we shall employ a stochastic comparison for functionals of the jump process $\left(\alpha_{t}\right)_{t \geq 0}$. More precisely,

Lemma 3.1 Assume (Q1) and (Q2) hold, and further $q_{i j}(x)=0$ for every $i, j \in \mathcal{M}$ with $|i-j| \geq 2$ and every $x \in \mathbb{R}^{3}$. For every $i, j \in \mathcal{M}$, let

$$
q_{i j}^{*}=\left\{\begin{array}{ll}
\sup _{x \in \mathbb{R}^{3}} q_{i j}(x), & j<i \\
\inf _{x \in \mathbb{R}^{3}} q_{i j}(x), & j>i \\
-\sum_{i \neq j} q_{i j}^{*}, & j=i
\end{array} \quad \text { and } \quad \bar{q}_{i j}= \begin{cases}\inf _{x \in \mathbb{R}^{3}} q_{i j}(x), & j<i \\
\sup _{x \in \mathbb{R}^{3}} q_{i j}(x), & j>i . \\
-\sum_{j \neq i} \bar{q}_{i j}, & j=i\end{cases}\right.
$$

Suppose that $\left(q_{i j}^{*}\right)$ and $\left(\bar{q}_{i j}\right)$ are irreducible and satisfy

$$
\begin{aligned}
& q_{i, i+1}(x)+q_{i+1, i}(x) \text { is independent of } x \text { for } 1 \leq i \leq N-2, \\
& \bar{q}_{N-1, N}+\bar{q}_{N, N-1} \leq q_{N-1, N}(x)+q_{N, N-1}(x), \quad \forall x \in \mathbb{R}^{3}, \\
& q_{N-1, N}^{*}+q_{N, N-1}^{*} \geq q_{N-1, N}(x)+q_{N, N-1}(x), \quad \forall x \in \mathbb{R}^{3} .
\end{aligned}
$$

Then, there exist two continuous-time Markov chains $\left(\alpha_{t}^{*}\right)_{t \geq 0}$ and $\left(\bar{\alpha}_{t}\right)_{t \geq 0}$ on $\mathcal{M}$ with transition rate matrix $\left(q_{i j}^{*}\right)$ and $\left(\bar{q}_{i j}\right)$ respectively such that for every nondecreasing function $\phi: \mathcal{M} \rightarrow \mathbb{R}_{+}$,

$$
\int_{0}^{t} \phi\left(\alpha_{s}^{*}\right) \mathrm{d} s \leq \int_{0}^{t} \phi\left(\alpha_{s}\right) \mathrm{d} s \leq \int_{0}^{t} \phi\left(\bar{\alpha}_{s}\right) \mathrm{d} s, \quad \text { a.s. }
$$

Proof. One can follow the idea of the argument to show [24, Lemma 2.8] to prove this lemma, although only the upper bound is proved therein. So the proof of this lemma is omitted to save space.

Under the condition that $\left(q_{i j}^{*}\right)$ and $\left(\bar{q}_{i j}\right)$ are irreducible, the finiteness of $\mathcal{M}$ yields that $\left(\alpha_{t}^{*}\right)_{t \geq 0}$ and $\left(\bar{\alpha}_{t}\right)_{t \geq 0}$ are positive recurrent. Let $\pi^{*}=\left(\pi_{1}^{*}, \pi_{2}^{*}, \cdots, \pi_{M}^{*}\right)$ and $\bar{\pi}=\left(\bar{\pi}_{1}, \bar{\pi}_{2}, \cdots, \bar{\pi}_{M}\right)$ 
be the invariant probability measures of the continuous-time Markov chains $\left(\alpha_{t}^{*}\right)_{t \geq 0}$ and $\left(\bar{\alpha}_{t}\right)_{t \geq 0}$, respectively, provided that both $\left(\alpha_{t}^{*}\right)_{t \geq 0}$ and $\left(\bar{\alpha}_{t}\right)_{t \geq 0}$ are irreducible and positive recurrent.

As an application of Lemma 3.1, we provide some sufficient conditions to judge the extinction of the infectious individuals for the SIRS model determined by (1.3) and (1.5).

Theorem 3.2 Assume that the assumptions of Theorem 2.5 and Lemma 3.1 hold. Suppose further that $i \mapsto \Gamma_{i}:=\Upsilon_{i}-\left(\mu_{i}+\nu_{i}+\delta_{i}\right)$ is nondecreasing and that

$$
\Theta_{5}:=\frac{\sum_{i \in \mathcal{M}} \bar{\pi}_{i} \Upsilon_{i}}{\sum_{i \in \mathcal{M}} \bar{\pi}_{i}\left(\mu_{i}+\nu_{i}+\delta_{i}\right)}<1
$$

or that $i \mapsto \Gamma_{i}$ is nonincreasing and that

$$
\Theta_{6}:=\frac{\sum_{i \in \mathcal{M}} \pi_{i}^{*} \Upsilon_{i}}{\sum_{i \in \mathcal{M}} \pi_{i}^{*}\left(\mu_{i}+\nu_{i}+\delta_{i}\right)}<1
$$

Then

$$
\lim _{t \rightarrow \infty} I_{t}=0, \text { a.s. and } \lim _{t \rightarrow \infty} R_{t}=0, \text { a.s. }
$$

Moreover, if $i \mapsto \Gamma_{i}$ is nondecreasing, then

$$
\sum_{i \in \mathcal{M}} \pi_{i}^{*} \Lambda_{i} \leq \liminf _{t \rightarrow \infty}\left(\int_{0}^{t} \mu_{\alpha_{s}} S_{s} \mathrm{~d} s\right) \leq \limsup _{t \rightarrow \infty}\left(\int_{0}^{t} \mu_{\alpha_{s}} S_{s} \mathrm{~d} s\right) \leq \sum_{i \in \mathcal{M}} \bar{\pi}_{i} \Lambda_{i} ;
$$

or if $i \mapsto \Gamma_{i}$ is nondecreasing, then

$$
\sum_{i \in \mathcal{M}} \bar{\pi}_{i} \Lambda_{i} \leq \liminf _{t \rightarrow \infty}\left(\int_{0}^{t} \mu_{\alpha_{s}} S_{s} \mathrm{~d} s\right) \leq \limsup _{t \rightarrow \infty}\left(\int_{0}^{t} \mu_{\alpha_{s}} S_{s} \mathrm{~d} s\right) \leq \sum_{i \in \mathcal{M}} \pi_{i}^{*} \Lambda_{i} .
$$

Proof. Once $\lim _{t \rightarrow \infty} I_{t}=0$, a.s., is available, $\lim _{t \rightarrow \infty} R_{t}=0$, a.s., can be proved similarly by following the trick in the argument of Theorem 2.5. So, in what follows, it remains to show that $\lim _{t \rightarrow \infty} I_{t}=0$, a.s. Observe that (2.9) still holds for the present setup. So, taking the nondecreasing property of $i \mapsto \Gamma_{i}$ as well as (3.3) and employing Lemma 3.1 and the ergodic theorem for the continuous-time Markov chains, we obtain the desired assertions (3.5).

If $i \mapsto \Gamma_{i}$ is nonincreasing, then $i \mapsto-\Gamma_{i}$ is nondecreasing trivially. So, an application of Lemma 3.1 yields that

$$
-\int_{0}^{t} \Gamma_{\alpha_{s}^{*}} \mathrm{~d} s \leq-\int_{0}^{t} \Gamma_{\alpha_{s}} \mathrm{~d} s \leq-\int_{0}^{t} \Gamma_{\bar{\alpha}_{s}} \mathrm{~d} s
$$

which further results in

$$
\int_{0}^{t} \Gamma_{\bar{\alpha}_{s}} \mathrm{~d} s \leq \int_{0}^{t} \Gamma_{\alpha_{s}} \mathrm{~d} s \leq \int_{0}^{t} \Gamma_{\alpha_{s}^{*}} \mathrm{~d} s
$$


Whence, the assertion (3.5) follows from (3.4) and the ergodic theorem for the continuous-time Markov chains.

By virtue of (2.2), it holds that

$$
\frac{1}{t} \int_{0}^{t} \mu_{\alpha_{t}} S_{s} \mathrm{~d} s=\frac{N_{0}-N_{t}}{t}+\frac{1}{t} \int_{0}^{t}\left\{\Lambda_{\alpha_{s}}-\left(\mu_{\alpha_{s}}+\delta_{\alpha_{s}}\right) I_{s}-\mu_{\alpha_{s}} R_{s}\right\} \mathrm{d} s .
$$

Thereby, (3.6) and (3.7) follow from (2.3), (3.5), Lemma 3.1, as well as the strong ergodic theorem for continuous-time Markov chains.

Hereinafter, two examples are set to show the applications of Theorem 3.2 .

Example 3.1 Consider the model (1.3) with $G$ introduced in (2.14), where $\left(\alpha_{t}\right)_{t \geq 0}$ is a continuoustime jump process with the state space $\mathcal{M}=\{1,2\}$ and the $Q$-matrix $Q(x)$ given by

$$
Q(x)=\left(\begin{array}{cc}
\sin x-p & p-\sin x \\
q+\sin x & -q-\sin x
\end{array}\right)
$$

Assume that

$$
\begin{aligned}
\Lambda_{2}<\Lambda_{1}, & \mu_{2}<\mu_{1}, \\
\beta_{1} \Lambda_{1} / \mu_{2}-\left(\mu_{1}+\nu_{1}+\delta_{1}\right)<0, & \beta_{2}\left(\Lambda_{1} / \mu_{2}\right)^{2}-\left(\mu_{2}+\nu_{2}+\delta_{2}\right)>0
\end{aligned}
$$

and that

$$
\frac{q-1}{1+p}>\frac{\beta_{2}\left(\Lambda_{1} / \mu_{2}\right)^{2}-\left(\mu_{2}+\nu_{2}+\delta_{2}\right)}{\mu_{1}+\nu_{1}+\delta_{1}-\beta_{1} \Lambda_{1} / \mu_{2}}
$$

A straightforward calculation shows that

$$
\bar{Q}=\left(\bar{q}_{i j}\right)_{1 \leq i, j \leq 2}=\left(\begin{array}{cc}
-(p+1) & p+1 \\
q-1 & 1-q
\end{array}\right) .
$$

Thus, the unique invariant probability measure of the continuous-time Markov chain $\left(\bar{\alpha}_{t}\right)$ generated by $\bar{Q}$ above is

$$
\bar{\pi}=\left(\frac{q-1}{p+q}, \frac{p+1}{p+q}\right) .
$$

By (3.8), one has $\check{\Lambda}=\Lambda_{1}$ and $\hat{\mu}=\mu_{2}$, which, together with (2.16), leads to $\Upsilon_{i}=\beta_{i}\left(\Lambda_{1} / \mu_{2}\right)^{i}$. On the other hand, (3.9) implies that $i \mapsto \Gamma_{i}=\Upsilon_{i}-\left(\mu_{i}+\nu_{i}+\delta_{i}\right)$ is nondecreasing. Furthermore, combining (3.10) with (1.4) ensures $\Theta_{5}$, defined in (3.3), is less than 1. Thus, the assertions (3.5) and (3.6) in Theorem 3.2 hold true. 
Example 3.2 We continue to investigate the model (1.3) with $G$ introduced in (2.14), where $\left(\alpha_{t}\right)_{t \geq 0}$ is a continuous-time jump process with the state space $\mathcal{M}=\{1,2\}$ and the $Q$-matrix $Q(x)$ set by

$$
Q(x)=\left(\begin{array}{cc}
\frac{x^{2}}{1+x^{2}}-p & p-\frac{x^{2}}{1+x^{2}} \\
q+\frac{x^{2}}{1+x^{2}} & -q-\frac{x^{2}}{1+x^{2}}
\end{array}\right) .
$$

In addition to (3.8), we further suppose that

$$
\beta_{1} \Lambda_{1} / \mu_{2}-\left(\mu_{1}+\nu_{1}+\delta_{1}\right)>0, \quad \beta_{2}\left(\Lambda_{1} / \mu_{2}\right)^{2}-\left(\mu_{2}+\nu_{2}+\delta_{2}\right)<0
$$

and that

$$
\frac{q+1}{p-1}<\frac{\mu_{2}+\nu_{2}+\delta_{2}-\beta_{2}\left(\Lambda_{1} / \mu_{2}\right)^{2}}{\beta_{1} \Lambda_{1} / \mu_{2}-\left(\mu_{1}+\nu_{1}+\delta_{1}\right)}
$$

Observe that

$$
Q^{*}=\left(q_{i j}^{*}\right)_{1 \leq i, j \leq 2}=\left(\begin{array}{cc}
p-1 & p-1 \\
q+1 & -q-1
\end{array}\right) .
$$

Then, the continuous-time Markov chain $\left(\alpha_{t}^{*}\right)$ generated by the $Q$-matrix $Q^{*}$ above possesses a unique invariant probability measure

$$
\pi^{*}=\left(\frac{q+1}{p+q}, \frac{p-1}{p+q}\right) .
$$

For the present setup, observe that $\Upsilon_{i}=\beta_{i}\left(\Lambda_{1} / \mu_{2}\right)^{i}$. From (3.12), we deduce that $i \mapsto \Gamma_{i}=$ $\Upsilon_{i}-\left(\mu_{i}+\nu_{i}+\delta_{i}\right)$ is nonincreasing. Moreover, (3.13) and (3.14) guarantee that $\Theta_{6}$, introduced in (3.4), is smaller than 1. Hence, we can make a conclusion that the assertions (3.5) and (3.7) hold by virtue of Theorem 3.2 .

For another application of Lemma 3.1, the following theorem provides a criterion to determine the persistence of the infectious individuals modelled by (1.3) and (1.5).

Theorem 3.3 Assume the assumptions of Theorem 2.8 and Lemma 3.1 hold. Suppose further that $i \mapsto \Gamma_{i}:=\Lambda_{i}-\left(\mu_{i}+\nu_{i}+\delta_{i}\right)$ is nondecreasing and that

$$
\Theta_{7}:=\frac{\sum_{i \in \mathcal{M}} \pi_{i}^{*} \Lambda_{i}}{\tau \sum_{i \in \mathcal{M}} \pi_{i}^{*}\left(\mu_{i}+\nu_{i}+\delta_{i}\right)}>1,
$$

where $\tau>0$ is introduced in (2.27); or that $i \mapsto \Theta_{i}$ is nonincreasing and that

$$
\Theta_{8}:=\frac{\sum_{i \in \mathcal{M}} \bar{\pi}_{i} \Lambda_{i}}{\tau \sum_{i \in \mathcal{M}} \bar{\pi}_{i}\left(\mu_{i}+\nu_{i}+\delta_{i}\right)}>1 .
$$

Then

$$
\liminf _{t \rightarrow \infty}\left(\frac{1}{t} \int_{0}^{t} I_{s} \mathrm{~d} s\right)>0, \quad \text { a.s. }
$$


Proof. We remark that (2.33) still holds for the present framework. Then, applying Lemma 3.1 and strong ergodic theorem for the continuous-time Markov chains and taking the nondecreasing (resp. nonincreasing) property of $i \mapsto \Gamma_{i}$ and (3.15) (resp. (3.16)) into consideration yields the desired assertion (3.17).

\section{Extension to stochastic SIRS}

In this section, we move forward to extend the random SIRS model (1.3) and (1.4) (or (1.5)) with a specific $G$ into the stochastic SIRS model determined by (1.6) and (1.5). Throughout this section, we still let $N_{t}=S_{t}+I_{t}+R_{t}$ and $\Psi_{i}=\mu_{i}+\nu_{i}+\delta_{i}+\left(\mu_{i}^{e}\right)^{2} / 2, i \in \mathcal{M}$.

Our main result in this section is stated as follows, which provides some sufficient conditions to examine the extinction of the infectious individuals.

Theorem 4.1 Assume the assumption (A3) and the conditions of Lemma 3.1 hold. If $i \mapsto$ $\Gamma_{i}:=\frac{\breve{\beta}^{e}}{\hat{\mu}} \Lambda_{i}-\Psi_{i}$ is nondecreasing and that

$$
\Theta_{9}:=\frac{\sum_{i \in \mathcal{M}} \bar{\pi}_{i} \Gamma_{i}}{\sum_{i \in \mathcal{M}} \bar{\pi}_{i}\left(\mu_{i}+\nu_{i}+\delta_{i}\right)}<1
$$

or that $i \mapsto \Gamma_{i}$ is nonincreasing and that

$$
\Theta_{10}:=\frac{\sum_{i \in \mathcal{M}} \pi_{i}^{*} \Gamma_{i}}{\sum_{i \in \mathcal{M}} \pi_{i}^{*}\left(\mu_{i}+\nu_{i}+\delta_{i}\right)}<1 .
$$

Then

$$
\lim _{t \rightarrow \infty} I_{t}=0 \quad \text { a.s. } \quad \lim _{t \rightarrow \infty} R_{t}=0
$$

Moreover, if $i \mapsto \Lambda_{i}$ is nondecreasing, then

$$
\sum_{i \in \mathcal{M}} \pi_{i}^{*} \Lambda_{i} \leq \liminf _{t \rightarrow \infty}\left(\int_{0}^{t} \mu_{\alpha_{s}} S_{s} \mathrm{~d} s\right) \leq \limsup _{t \rightarrow \infty}\left(\int_{0}^{t} \mu_{\alpha_{s}} S_{s} \mathrm{~d} s\right) \leq \sum_{i \in \mathcal{M}} \bar{\pi}_{i} \Lambda_{i}
$$

or if $i \mapsto \Lambda_{i}$ is nondecreasing, then

$$
\sum_{i \in \mathcal{M}} \bar{\pi}_{i} \Lambda_{i} \leq \liminf _{t \rightarrow \infty}\left(\int_{0}^{t} \mu_{\alpha_{s}} S_{s} \mathrm{~d} s\right) \leq \limsup _{t \rightarrow \infty}\left(\int_{0}^{t} \mu_{\alpha_{s}} S_{s} \mathrm{~d} s\right) \leq \sum_{i \in \mathcal{M}} \pi_{i}^{*} \Lambda_{i} .
$$

Before we proceed to complete the proof of Theorem 4.1, we prepare some auxiliary lemmas. 
Lemma 4.2 Assume that (A3) holds. Then, (1.6) and (1.5) has a unique strong solution $\left(S_{t}, I_{t}, R_{t}\right) \in \mathbb{R}_{+}^{3}$ for the initial value $\left(s_{0}, i_{0}, r_{0}\right) \in \mathbb{R}_{+}^{3}$. Moreover, for any $p \in\left(1,1+2 \hat{\mu} /\left(\check{\mu}^{e}\right)^{2}\right)$ there exists a constant $C_{p}>0$ such that

$$
\sup _{k \in \mathbb{N}} \mathbb{E}\left(\sup _{k \leq s \leq k+1} N_{s}^{p}\right) \leq C_{p}
$$

Proof. By following the argument of Lemma 2.2 and making use of the Lyapunov function for any $x=\left(x_{1}, x_{2}, x_{3}\right) \in \mathbb{R}_{+}^{3}$,

$$
V(x)=\left(x_{1}+x_{2}+x_{3}\right)^{2}+x_{1}-a-a \ln \left(x_{1} / a\right)+x_{2}-1-\ln x_{2}+x_{3}-1-\ln x_{3}
$$

for some constant $a>0$ chosen suitably, we conclude that (1.6) and (1.5) has a unique strong solution $\left(S_{t}, I_{t}, R_{t}\right) \in \mathbb{R}_{+}^{3}$ for the initial value $\left(s_{0}, i_{0}, r_{0}\right) \in \mathbb{R}_{+}^{3}$.

From (1.6), it is obvious to see that

$$
\mathrm{d} N_{t}=\left\{\Lambda_{\alpha_{t}}-\mu_{\alpha_{t}} N_{t}-\delta_{\alpha_{t}} I_{t}\right\} \mathrm{d} t-\mu_{\alpha_{t}}^{e} N_{t} \mathrm{~d} B_{t}^{(1)}, \quad t \geq 0
$$

In what follows, we fix $p \in\left(1,\left(1+2 \hat{\mu} /\left(\check{\mu}^{e}\right)^{2}\right)\right]$. By Itô's formula, it follows that

$$
\mathrm{d} N_{t}^{p}=p N_{t}^{p-1}\left\{\Lambda_{\alpha_{t}}-\left(\mu_{\alpha_{t}}-(p-1)\left(\mu_{\alpha_{t}}^{e}\right)^{2} / 2\right) N_{t}-\delta_{\alpha_{t}} I_{t}\right\} \mathrm{d} t-p \mu_{\alpha_{t}}^{e} N_{t}^{p} \mathrm{~d} B_{t}^{(1)} .
$$

Taking advantage of $I_{t} \geq 0$ and Young's inequality: $a^{\alpha} b^{1-\alpha} \leq \alpha a+(1-\alpha) b, a, b \geq 0, \alpha \in(0,1)$, yields that

$$
\begin{aligned}
\mathbb{E} N_{t}^{p} & \left.\leq \mathbb{E} N_{s}^{p}+\int_{s}^{t}\left\{p \check{\Lambda} \mathbb{E} N_{u}^{p-1}-\left(\hat{\mu}-(p-1)\left(\check{\mu}^{e}\right)^{2} / 2\right)\right) \mathbb{E} N_{u}^{p}\right\} \mathrm{d} u \\
& \leq \mathbb{E} N_{s}^{p}+\int_{s}^{t}\left\{c_{1}-c_{2} \mathbb{E} N_{u}^{p}\right\} \mathrm{d} u, \quad t \geq s \geq 0, p \in\left(1,1+2 \hat{\mu} /\left(\check{\mu}^{e}\right)^{2}\right)
\end{aligned}
$$

for some constants $c_{1}, c_{2}>0$. Subsequently, Gronwall's inequality gives that for some $c_{3}>0$,

$$
\sup _{t \geq 0} \mathbb{E} N_{t}^{p} \leq c_{3} .
$$

Moreover, by BDG's inequality and Young's inequality, we deduce from (4.8) that there exist constants $c_{4}, c_{5}>0$ such that for any $t \in[k, k+1]$,

$$
\begin{aligned}
\mathbb{E}\left(\sup _{k \leq s \leq t} N_{s}^{p}\right) & \leq \mathbb{E} N_{k}^{p}+c_{4} \int_{k}^{t} \mathbb{E} N_{s}^{p} \mathrm{~d} s+c_{4} \mathbb{E}\left(\int_{k}^{t} N_{s}^{2 p} \mathrm{~d} s\right)^{1 / 2} \\
& \leq \mathbb{E} N_{k}^{p}+c_{4} \int_{k}^{t} \mathbb{E} N_{s}^{p} \mathrm{~d} s+c_{4} \mathbb{E}\left(\sup _{k \leq s \leq t} N_{s}^{p} \int_{k}^{t} N_{s}^{p} \mathrm{~d} s\right)^{1 / 2} \\
& \leq \frac{1}{2} \mathbb{E}\left(\sup _{k \leq s \leq t} N_{s}^{p}\right)+\mathbb{E} N_{k}^{p}+c_{5} \int_{k}^{t} \mathbb{E} N_{s}^{p} \mathrm{~d} s
\end{aligned}
$$

This, combining with (4.9), yields (4.6). 
Lemma 4.3 Under the assumption (A3),

$$
\lim _{t \rightarrow \infty}\left(\frac{1}{t} \int_{0}^{t} \frac{\beta_{\alpha_{s}}^{e} S_{s}}{f\left(I_{s}\right)} \mathrm{d} B_{s}^{(2)}\right)=0 \quad \text { a.s. }
$$

and, for $M_{t}:=\mu_{\alpha_{s}} N_{t}+\delta_{\alpha_{s}} I_{t}-\Lambda_{\alpha_{t}}$,

$$
\lim _{t \rightarrow \infty}\left(\frac{1}{t} \int_{0}^{t} M_{s} \mathrm{~d} s\right)=0, \quad \text { a.s. }
$$

Proof. To derive (4.10), it suffices to verify that

$$
\lim _{k \rightarrow \infty}\left(\frac{1}{k} \sup _{t \in[k, k+1]}\left|\int_{k}^{t} \frac{\beta_{\alpha_{s}}^{e} S_{s}}{f\left(I_{s}\right)} \mathrm{d} B_{s}^{(2)}\right|\right)=0 \text { a.s. }
$$

and

$$
\lim _{k \rightarrow \infty}\left(\frac{1}{k} \int_{0}^{k} \frac{\beta_{\alpha_{s}}^{e} S_{s}}{f\left(I_{s}\right)} \mathrm{d} B_{s}^{(2)}\right)=0 \quad \text { a.s. }
$$

Hereinafter, we stipulate $p \in\left(1,\left(1+2 \hat{\mu} /\left(\check{\mu}^{e}\right)^{2}\right) \wedge 2\right)$. By BDG's inequality, we deduce from (4.6) and (A3) that there exist constants $\tilde{C}_{p}, \hat{C}_{p}>0$ such that

$$
\begin{aligned}
\mathbb{E}\left(\sup _{t \in[k, k+1]}\left|\int_{k}^{t} \frac{\beta_{\alpha_{s}}^{e} S_{s}}{f\left(I_{s}\right)} \mathrm{d} B_{s}^{(2)}\right|^{p}\right) & \leq \frac{\tilde{C}_{p}\left(\check{\beta}^{e}\right)^{p}}{f^{p}(0)} \mathbb{E}\left(\int_{k}^{k+1} S_{s}^{2} \mathrm{~d} s\right)^{p / 2} \\
& \leq \frac{\tilde{C}_{p}\left(\check{\beta}^{e}\right)^{p}}{f^{p}(0)} \mathbb{E}\left(\sup _{k \leq s \leq k+1} S_{s}^{p}\right) \leq \hat{C}_{p} .
\end{aligned}
$$

For any $M>0$ and each integer $k \geq 1$, set

$$
A_{k, M}:=\left\{\frac{1}{k} \sup _{t \in[k, k+1]}\left|\int_{k}^{t} \frac{\beta_{\alpha_{s}}^{e} S_{s}}{f\left(I_{s}\right)} \mathrm{d} B_{s}^{(2)}\right| \geq M\right\} .
$$

Via Chebyshev's inequality, it follows from (4.14) that

$$
\mathbb{P}\left(A_{k, M}\right) \leq \frac{1}{k^{p} M^{p}} \mathbb{E}\left(\sup _{t \in[k, k+1]}\left|\int_{k}^{t} \frac{\beta_{\alpha_{s}}^{e} S_{s}}{f\left(I_{s}\right)} \mathrm{d} B_{s}^{(2)}\right|^{p}\right) \leq \frac{\hat{C}_{p}}{k^{p} M^{p}},
$$

Since the series $\sum_{k=1}^{\infty} 1 / k^{p}$ converges, by the Borel-Cantelli lemma, we can conclude that

$$
\mathbb{P}\left(\limsup _{k \rightarrow \infty} A_{k, M}\right)=0
$$

Therefore, (4.12) follows due to the arbitrariness of $M$. 
Now we turn to claim that (4.13) holds. Let

$$
s_{0}=0, \quad s_{k}=\int_{0}^{k} \frac{\beta_{\alpha_{s}}^{e} S_{s}}{f\left(I_{s}\right)} \mathrm{d} B_{s}^{(2)}, \quad x_{k}=s_{k}-s_{k-1}, \quad k \geq 1 .
$$

Clearly, $\mathbb{E}\left(x_{k} / k\right)<\infty$. On the other hand, (4.14) implies that

$$
\sum_{k=1}^{\infty} \mathbb{E}\left(\left|x_{k}\right|^{p} \mid \mathscr{F}_{k-1}\right) k^{-p} \leq \hat{C}_{p} \sum_{k=1}^{\infty} k^{-p}<\infty .
$$

Thus, (4.13) is available from [6, Theorem 5].

Following the arguments to derive (4.12) and (4.13), respectively, we deduce that

$$
\lim _{t \rightarrow \infty} \frac{N_{t}}{t}=0, \quad \text { a.s. } \quad \lim _{t \rightarrow \infty} \frac{1}{t}\left(\int_{0}^{t} \mu_{\alpha_{s}}^{e} N_{s} \mathrm{~d} B_{s}^{(1)}\right)=0, \quad \text { a.s. }
$$

Those, together with (4.7), yields (4.11).

With Lemmas 4.3 and 4.2 in hand, we are now in position to finish the

Proof of Theorem 4.1 For notation simplicity, let

$$
I_{1}(t)=\frac{\check{\beta}^{e}}{\hat{\mu} f(0)} \cdot \frac{1}{t} \int_{0}^{t} M_{t} \mathrm{~d} t, \quad I_{2}(t)=-\int_{0}^{t} \mu_{\alpha_{s}}^{e} \mathrm{~d} B_{s}^{(1)}, \quad I_{3}(t)=\frac{1}{t} \int_{0}^{t} \frac{\beta_{\alpha_{s}}^{e} S_{s}}{f\left(I_{s}\right)} \mathrm{d} B_{s}^{(2)} .
$$

By the Itô formula, we find from $(\mathbf{A} 3)$ and $0 \leq S_{t} \leq N_{t}$ that

$$
\begin{aligned}
\frac{1}{t} \ln \left(I_{t} / I_{0}\right) & =\frac{1}{t} \int_{0}^{t}\left\{\frac{\beta_{\alpha_{s}}^{e} S_{s}}{f\left(I_{s}\right)}-\frac{1}{2} \frac{\left(\beta_{\alpha_{s}}^{e}\right)^{2} S_{s}^{2}}{f\left(I_{s}\right)^{2}}-\Psi_{\alpha_{s}}\right\} \mathrm{d} s+\frac{I_{2}(t)}{t}+I_{3}(t) \\
& \leq \frac{1}{t} \int_{0}^{t}\left\{\beta_{\alpha_{s}}^{e} S_{s} / f(0)-\Psi_{\alpha_{s}}\right\} \mathrm{d} s+\frac{I_{2}(t)}{t}+I_{3}(t) \\
& \leq I_{1}(t)+\frac{1}{t} \int_{0}^{t}\left\{\frac{\check{\beta}^{e}}{\hat{\mu} f(0)} \Lambda_{\alpha_{s}}-\Psi_{\alpha_{s}}\right\} \mathrm{d} s+\frac{I_{2}(t)}{t}+I_{3}(t) .
\end{aligned}
$$

By (4.10) and (4.11), one has

$$
\lim _{t \rightarrow \infty}\left(I_{1}(t)+I_{3}(t)\right)=0, \quad \text { a.s. }
$$

Let $\left\langle I_{2}\right\rangle_{t}$ be the quadratic variation of $I_{2}(t)$. A simple calculation shows that

$$
\limsup _{t \rightarrow \infty} \frac{1}{t}\left\langle I_{2}\right\rangle_{t} \leq\left(\check{\mu}^{e}\right)^{2}
$$

so that the strong law of large numbers for continuous martingales gives that

$$
\lim _{t \rightarrow \infty} \frac{1}{t} I_{2}(t)=0, \quad \text { a.s. }
$$


Combining (4.17) with (4.18) and employing $i \mapsto \Gamma_{i}$ is nondecreasing (resp. nonincreasing) and (4.1) (resp. (4.2)), we deduce from Lemma 3.1 and the strong ergodic theorem for continuoustime Markov chains that

$$
\lim _{t \rightarrow \infty} I_{t}=0 \quad \text { a.s. }
$$

which implies that for any $\varepsilon \in(0,1)$ sufficiently small there exist $\Omega_{1} \subseteq \Omega$ with $\mathbb{P}\left(\Omega_{1}\right)=1$ and $T_{1}=T_{1}(\omega)>0$ such that

$$
I_{t}(\omega) \leq \varepsilon, \quad t \geq T_{1}, \quad \omega \in \Omega_{1} .
$$

Set $\xi_{i}:=\mu_{i}+\gamma_{i}+\left(\mu_{i}^{e}\right)^{2} / 2, i \in \mathcal{M}$, and for any $0 \leq s \leq t$,

$$
\Lambda_{s, t}:=\int_{s}^{t} \mu_{\alpha_{u}}^{e} \mathrm{~d} B_{u}^{(1)} \quad \text { and } \quad \Phi_{s, t}:=\exp \left(-\int_{s}^{t} \xi_{\alpha_{u}} \mathrm{~d} u-\Lambda_{s, t}\right) .
$$

By (4.18), we have

$$
\lim _{t \rightarrow \infty} \frac{1}{t} \ln \Phi_{0, t}<0, \quad \text { a.s. }
$$

which implies that there exists $\Omega_{2} \subseteq \Omega$ with $\mathbb{P}\left(\Omega_{2}\right)=1$ and $T_{2}=T_{2}(\omega)>0$ such that

$$
\Phi_{0, t}(\omega) \leq \varepsilon, \quad t \geq T_{2}, \quad \omega \in \Omega_{2}
$$

Next, using the law of the iterated logarithm for stochastic integrals [27, (1.2)], we deduce that

$$
\liminf _{t \rightarrow \infty} \frac{\Lambda_{s, t}}{\sqrt{2\langle\Lambda\rangle_{s, t} \ln \ln \langle\Lambda\rangle_{s, t}}}=-1 \quad \text { a.s. }
$$

So there exists $\Omega_{3} \subseteq \Omega$ with $\mathbb{P}\left(\Omega_{3}\right)=1, T_{3}=T_{3}(\omega)>0$ such that

$$
-(1+\varepsilon) \sqrt{2\langle\Lambda\rangle_{s, t} \ln \ln \langle\Lambda\rangle_{s, t}} \leq \Lambda_{s, t} \leq(-1+\varepsilon) \sqrt{2\langle\Lambda\rangle_{s, t} \ln \ln \langle\Lambda\rangle_{s, t}}, \quad t, s \geq T_{2}, \quad \varepsilon \in \Omega_{3} .
$$

By the variation-of-constants formula (see e.g. [20, Theorem 3.1]), we deduce from (4.19) and (4.21) that for any $\omega \in \Omega_{0}:=\Omega_{1} \cap \Omega_{2} \cap \Omega_{3}$ and $t \geq T:=T_{1}+T_{2}+T_{3}$,

$$
\begin{aligned}
R_{t}(\omega)= & \Phi_{0, t}(\omega)\left\{R_{0}+\int_{0}^{t} \nu_{\alpha_{s}}(\omega) I_{s}(\omega) \Phi_{0, s}^{-1}(\omega) \mathrm{d} s\right\} \\
\leq & R_{0} \Phi_{0, t}(\omega)+\check{\nu} \Phi_{T, t}(\omega) \int_{0}^{T} I_{s}(\omega) \Phi_{s, T}(\omega) \mathrm{d} s+\check{\nu} \int_{T}^{t} I_{s}(\omega) \Phi_{s, t}(\omega) \mathrm{d} s \\
\leq & R_{0} \varepsilon+\check{\nu} \varepsilon \int_{T}^{t} \exp \left(-\int_{s}^{t} \xi_{\alpha_{u}} \mathrm{~d} u+(1+\varepsilon) \sqrt{2\langle\Lambda\rangle_{s, t} \ln \ln \langle\Lambda\rangle_{s, t}}\right) \mathrm{d} s \\
& +\check{\nu} \int_{0}^{T} I_{s}(\omega) \Phi_{s, T}(\omega) \mathrm{d} s \exp \left(-\int_{T}^{t} \xi_{\alpha_{u}} \mathrm{~d} u+(1+\varepsilon) \sqrt{2\langle\Lambda\rangle_{T, t} \ln \ln \langle\Lambda\rangle_{T, t}}\right) .
\end{aligned}
$$


Furthermore, observe that there exist constants $c>0$ and $\alpha \in(0,1)$ such that

$$
(1+\varepsilon) \sqrt{2\langle\Lambda\rangle_{s, t} \ln \ln \langle\Lambda\rangle_{s, t}} \leq c+\alpha \int_{s}^{t} \xi_{\alpha_{u}} \mathrm{~d} u
$$

Plugging (4.23) into (4.22) and making use of the arbitrariness of $\varepsilon \in(0,1)$, we obtain (4.3).

With (4.3) in hand, we deduce from (4.7) and (4.16) that

$$
\lim _{t \rightarrow \infty}\left(\frac{1}{t} \int_{0}^{t}\left(\Lambda_{\alpha_{s}}-\mu_{\alpha_{s}} S_{s}\right) \mathrm{d} s\right)=0
$$

This, combining with Lemma 3.1, yields the assertions (4.4) and (4.5).

Acknowledgement. This work is supported by NNSFs of China (Nos. 11771327, 11301030, 11431014, 11831014).

\section{References}

[1] Anderson, W.J., 1991. Continuous-Time Markov Chains. Springer, Berlin.

[2] Bao, J. Shao, J., Permanence and extinction of regime-switching predator-prey models, SIAM J. Math. Anal. 48 (2016), No. 1, 725-739.

[3] Cai, Y., Kang, Y., Banerjee, M., Wang, W., A stochastic SIRS epidemic model with infectious force under intervention strategies, J. Differential Equations, 259 (2015), 7463-7502.

[4] Capasso, Y., Serio, G., A generalization of the Kermack-Mckendrick deterministic epidemic model, Math. Biosci., 42 (1978), 43-61.

[5] Chen, M.-F.: Eigenvalues, inequalities, and Ergodicity Theory, Springer, London, 2005.

[6] Chow, Y. S. Local convergence of martingale and the law of large numbers, Ann. Math. Statist., 36 (1965), 552-558.

[7] Da Prato, G., Zabczyk, J., Ergodicity for Infinite Dimensional Systems, Cambridge Univ. Press, Cambridge, 1996.

[8] Dang, N. H., Du, N. H., Yin, G., Existence of stationary distributions for Kolmogorov systems of competitive type under telegraph noise, J. Differential Equations, 257 (2014), 2078-2101.

[9] Derrick, W. R., van den Driessche, P., A disease transmission model in a nonconstant population, J. Math. Biol., 31 (1993), 495-512.

[10] Du, N. H., Dang, N. H., Dynamics of Kolmogorov systems of competitive type under the telegraph noise, J. Differential Equations, 250 (2011), 386-409.

[11] Gray, A., Greenhalgh, D., Mao, X., Pan, J., The SIS epidemic model with Markovian switching, J. Math. Anal. Appl., 394 (2012), 496-516.

[12] Greenhalgh, D., Liang, Y., Mao, X., Modelling the effect of telegraph noise in the SIRS epidemic model using Markovian switching, Physica A, 462 (2016) 684-704.

[13] Hethcote, H., The mathematics of infectious diseases, SIAM Rev., 42 (2000), 599-653.

[14] Hieu, N. T., Du, N. H., Auger, P., Dang, N. H., Dynamical behavior of a stochastic SIRS epidemic model, Math. Model. Nat. Phenom., 10 (2015), 56-73. 
[15] Kermack, W. O., McKendrick, A. G., A contribution to the mathematical theory of epidemics, Proc. R. Soc. Lond. Ser. A, 115 (1927), 700-721.

[16] Lahrouz, A., Omari, L., Extinction and stationary distribution of a stochastic SIRS epidemic model with non-linear incidence, Statist. Probab. Lett., 83 (2013), 960-968.

[17] Li, D., Liu, S.,Cui, J., Threshold dynamics and ergodicity of an SIRS epidemic model with Markovian switching, J. Differential Equations, 263 (2017), 8873-8915.

[18] Li, X., Yin, G., Logistic models with regime switching: permanence and ergodicity, J. Math. Anal. Appl., 441 (2016), 593-611.

[19] Liu, W., Levin, S., Iwasa, Y., Influence of nonlinear incidence rate upon the behavior of SIRS epidemiological models, J. Math. Biol., 23 (1986), 187-204.

[20] Mao, X., Stochastic Differential Equations and Applications, Horwood, England, Second Edition, 2008.

[21] Mao, X., Yuan, C., Stochastic Differential Equations with Markovian Switching, Imperial College Press, London, 2006.

[22] N'zi, M., Kanga, G., Global analysis of a deterministic and stochastic nonlinear SIRS epidemic model with saturated incidence rate, Random Oper. Stoch. Equ., 2 (2016), 65-77.

[23] Shao, J., Strong solutions and strong Feller properties for regime-switching diffusion processes in an infinite state space, SIAM J. Control Optim., 53 (2015), 2462-2479

[24] Shao, J., Invariant measures and Euler-Maruyama's approximations of state-dependent regimeswitching diffusions, to appear in SIAM J. Control Optim., arXiv:1710.09168,

[25] Tang, Y., Huang, D., Ruan, S., Zhang, W., Coexistence of limit cycles and homoclinic loops in a SIRS model with a nonlinear incidence rate, SIAM J. Appl. Math., 69 (2008), 621-639.

[26] Tornatore, E., Buccellato, S. M., Vetro, P., Stability of a stochastic SIR system, Physica A, 354 (2005), 111-126.

[27] Wang, J.-G., A law of the iterated logarithm for stochastic integrals, Stochastic Process. Appl., 47 (1993), 215-228.

[28] Xi, F., Shao, J., Successful couplings for diffusion processes with state-dependent switching, Sci. China Math., 56 (2013), 2135-2144.

[29] Xiao, D., Ruan, S., Global analysis of an epidemic model with nonmonotone incidence rate, Math. Biosci., 208 (2007), 419-429.

[30] Yang, Q., Mao, X., Stochastic dynamics of SIRS epidemic models with random perturbation, Math. Biosci. Eng., 11 (2014), 1003-1025.

[31] Yin, G., Zhu, C., Hybrid switching diffusions: properties and applications, Vol. 63, Stochastic Modeling and Applied Probability, Springer, New York. 2010.

[32] Zhao, Y., Jiang, D., The threshold of a stochastic SIRS epidemic model with saturated incidence, Appl. Math. Lett., 34 (2014), 90-93. 